\title{
A peculiar planetary nebula candidate in a globular cluster in the Fornax dwarf spheroidal galaxy ${ }^{\star}$
}

\begin{abstract}
S. S. Larsen
Astronomical Institute, University of Utrecht, Princetonplein 5, 3584 CC Utrecht, The Netherlands e-mail: larsen@astro.uu.nl

Received 28 October 2007 / Accepted 12 November 2007

ABSTRACT

Context. Until now, only one planetary nebula (PN) has been known in the Fornax dwarf spheroidal galaxy.

Aims. The discovery of a second PN candidate, associated with one of the 5 globular clusters in the Fornax dwarf, is reported. Methods. Spectra of the globular cluster H5, obtained with the UVES echelle spectrograph on the ESO Very Large Telescope, show [O III] line emission at a radial velocity consistent with membership of the Fornax dwarf. A possible counterpart of the [O III] emission is identified in archival images from the Wide Field Planetary Camera 2 on board the Hubble Space Telescope. The source of the emission is located about 1 ." 5 (less than one core radius) southwest of the centre of the cluster.

Results. The emission line source is identified as a likely PN, albeit with several peculiar properties. No $\mathrm{H} \beta$, He I, or He II line emission is detected and the $[\mathrm{O} \mathrm{III}] / \mathrm{H} \beta$ ratio is $>25(2 \sigma)$. The expansion velocity inferred from the [O III] $5007 \AA$ line is $V_{\mathrm{e}} \approx 55 \mathrm{~km} \mathrm{~s}^{-1}$, which is large for a PN. The diameter measured on the HST images is about 0.23 or $0.15 \mathrm{pc}$ at the distance of the Fornax dSph.

Conclusions. This object doubles the number of known PNe in Fornax, and is only the 5th PN associated with an old GC for which direct imaging is available. It may be a member of the rare class of extremely H-deficient PNe, the second such case found in a GC.
\end{abstract}

Key words. galaxies: individual: Fornax dwarf spheroidal - galaxies: star clusters - stars: AGB and post-AGB planetary nebulae: individual: general

\section{Introduction}

Like many other dwarf spheroidal galaxies, the Fornax dwarf spheroidal (dSph) has a remarkably complex star formation history. Colour-magnitude diagrams show evidence of star formation until as recently as $\sim 10^{8}$ years ago (Stetson et al. 1998; Buonanno et al. 1999). The metallicity distribution of the field stars peaks at $[\mathrm{Fe} / \mathrm{H}] \sim-1.0$, but with tails extending up to nearly solar metallicity and down to $[\mathrm{Fe} / \mathrm{H}] \approx-2.7$ (Helmi et al. 2006). Battaglia et al. (2006) identified three more or less distinct stellar populations in the Fornax dSph, namely an old, metalpoor component, a dominant intermediate-age (2-8 Gyr) moderately metal-rich component, and a younger $(\sim 1 \mathrm{Gyr})$ metal-rich component.

Five globular clusters (GCs) are known in the Fornax dSph, catalogued by Hodge (1961) and hereafter referred to as H1...H5. When normalised to the (low) luminosity of the galaxy, this leads to an extremely high GC specific frequency of $S_{N} \approx 29$ (e.g. van den Bergh 1998). Historically, it is interesting to note that one of the GCs (NGC $1049=\mathrm{H} 3$ ) was already discovered by John Herschel nearly a century before the parent galaxy itself. From high-dispersion spectroscopy of individual stars in clusters $\mathrm{H} 1, \mathrm{H} 2$, and H3, Letarte et al. (2006) derived $[\mathrm{Fe} / \mathrm{H}]=-2.5 \pm 0.1,[\mathrm{Fe} / \mathrm{H}]=-2.1 \pm 0.1$, and $[\mathrm{Fe} / \mathrm{H}]=$ $-2.4 \pm 0.1$, respectively. From low-resolution spectroscopy, Strader et al. (2003) found an average $[\mathrm{Fe} / \mathrm{H}] \sim-1.8$ and old ages (comparable to Milky Way GCs), with H5 possibly a few

^ Based on observations collected at the European Southern Observatory, Chile under programme 078.B-0631(A), and with the NASA/ESA Hubble Space Telescope.
Gyrs younger than the rest. Thus, the GCs are most likely associated with the old, metal-poor component in Fornax.

The Fornax dSph is known to host a single planetary nebula (PN), discovered by Danziger et al. (1978). In a recent study of this object, Kniazev et al. (2007) derived an oxygen abundance of $12+\log (\mathrm{O} / \mathrm{H})=8.28 \pm 0.02([\mathrm{O} / \mathrm{H}]=-0.55$ for the solar oxygen abundance of Grevesse \& Sauval 1998). They also estimated an iron abundance of $[\mathrm{Fe} / \mathrm{H}]=-1.13 \pm 0.18$. It seems likely that this PN is associated with the intermediate-age component, but as for most $\mathrm{PNe}$, pinpointing the exact age (and hence the mass of the progenitor star) is difficult.

This Letter reports the discovery of a second PN candidate in the Fornax dSph, apparently associated with one of the GCs (H5). This not only doubles the number of known PNe in Fornax, but also constitutes a significant addition to the small number of PNe currently known to be associated with GCs in general (four in Milky Way GCs; Jacoby et al. 1997). The identification of PNe in stellar clusters holds special significance since it gives a better handle on the age and metallicity of the progenitor star (although binary star evolution may be an important path for PN formation in clusters). Furthermore, the Fornax dSph is close enough that spatially resolved imaging of the $\mathrm{PN}$ is within reach.

\section{Identification of a new PN candidate in $\mathrm{H} 5$}

\subsection{UVES spectroscopy}

Three of the Fornax GCs (H3, H4, and H5) were observed with the UVES echelle spectrograph on the ESO Very Large Telescope on Nov. 19 and Nov. 20, 2006. The primary aim of 


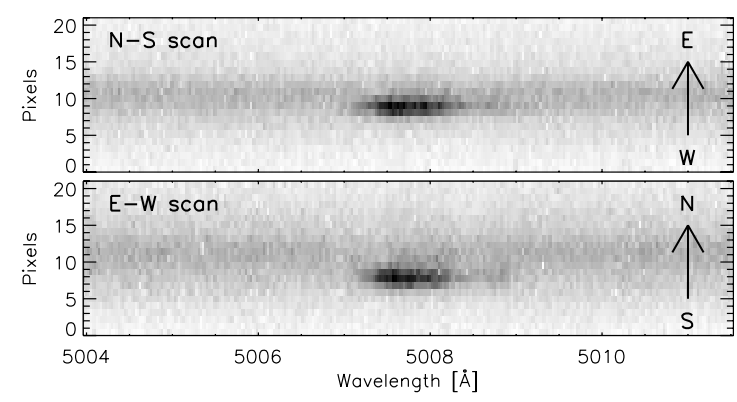

Fig. 1. Sections of the echellograms near the [O III] $5007 \AA ̊$ Aine.

the observations was detailed abundance analysis from the integrated cluster light, which will be described elsewhere. To sample the integrated light of the clusters, the UVES slit was scanned across each cluster in the east-west and north-south direction. In each direction, two scans of $2400 \mathrm{~s}$ each were obtained, which for $\mathrm{H} 5$ covered a range of $\pm 10^{\prime \prime}$ with respect to the cluster centre (the half-light radius of H5 is 9.'6; Mackey \& Gilmore 2003). The observations were carried out with the red arm alone, covering a wavelength interval of $4200 \AA-6200 \AA$ with a small gap near $5200 \AA$ between the two CCD detectors. The slit length and width were $8 . \prime 9$ and $11^{\prime \prime} 0$, respectively, yielding a spectral resolution of $\lambda / \Delta \lambda \approx 40000$. The observations were made under clear conditions with only occasional thin clouds.

Figure 1 shows the co-added east-west and north-south scans of $\mathrm{H} 5$ for the spectral region near $5007 \AA$. Each panel covers the full slit length of 8.9 with the arrows indicating the spatial direction. In addition to the stellar continuum from the cluster stars, the scans clearly show emission at $\sim 5008 \AA$, consistent with the wavelength of [O III] at the radial velocity of the Fornax dSph $\left(53 \mathrm{~km} \mathrm{~s}^{-1}\right.$, according to the NASA/IPAC Extragalactic Database). The emission is spatially unresolved in the UVES data and offset from the centre of the GC trace by about 3.5 pixels $\left(\sim 1^{\prime \prime} .4\right)$ towards the south and by about 2 pixels ( $\sim$.'8) towards the west. The source of the emission is thus located well within one core radius (2'. 1; Mackey \& Gilmore 2003) from the centre of H5.

Figure 2 shows one-dimensional spectra of the rows with the line emission for selected wavelength intervals. These spectra were constructed by co-adding the rows containing line emission and subtracting the sum of the remaining rows, scaled to the same mean level. An additional emission line, about 3 times fainter, is seen at $4960 \AA$, confirming the identification of the line emission as [O III]. Although the two oxygen lines are detected at $\sim 17 \sigma$ and $\sim 50 \sigma$, no other emission features are seen in the spectrum. In particular, there is no trace of either the $\mathrm{H} \beta$ line, the He I $4471 \AA$, or the He II $4686 \AA$ lines.

Since no flux standards were observed, an accurate flux calibration of the data is not possible and only crude constraints can be placed on line fluxes and ratios. The observed count ratio of the two [O III] lines is $3.4 \pm 0.2$, close to the theoretically expected intensity ratio of 3.0 (e.g. Osterbrock 1988). Only upper limits can be placed on the $[\mathrm{O} \mathrm{III}] / \mathrm{H} \beta$ ratio: since the [O III] $5007 \AA$ line is detected at about $50 \sigma$, a $2 \sigma$ lower limit is $[\mathrm{O} \mathrm{III}] / \mathrm{H} \beta>25$. This ratio only refers to uncalibrated counts, but since the separation in wavelength between $\mathrm{H} \beta$ and [O III] is modest, the corresponding limit on the flux ratio is probably not very different. Even this lower limit is a very high $[\mathrm{O} \mathrm{III}] / \mathrm{H} \beta$ ratio for a PN. The Acker et al. (1992) catalogue lists $[\mathrm{O}$ III $] / \mathrm{H} \beta$ line ratios for 886 Galactic $\mathrm{PNe}$, with a median $[\mathrm{O} \mathrm{III}] / \mathrm{H} \beta$ ratio of
8.9 and only about $3 \%$ of the $\mathrm{PNe}$ have $[\mathrm{O} \mathrm{III}] / \mathrm{H} \beta>20$. High $[\mathrm{O} \mathrm{III}] / \mathrm{H} \beta$ ratios usually imply a very high degree of excitation, but this should also be accompanied by strong He II lines (Dopita \& Meatheringham 1990), which however remain undetectable here. An alternative explanation for the lack of Balmer emission is depletion of hydrogen.

A few $\mathrm{K}$ giants that were observed during the run can be used to establish a crude flux calibration. By combining our observations of HD 83516 and HD 223094 with their $B$ magnitudes, a conversion between count rate and flux was defined at $\sim 4400 \AA$. Applying this conversion also at $5000 \AA$, the corresponding [O III] $5007 \AA$ line flux of the source in $\mathrm{H} 5$ is estimated to be $F_{5007} \approx 5 \times 10^{-15} \mathrm{erg} \mathrm{cm}^{-2} \mathrm{~s}^{-1}$ or $m_{5007} \approx 22$. The adopted conversion between count rate and flux corresponds to a total throughput of $\approx 10 \%$ (UVES + telescope + atmosphere) for an $8.2 \mathrm{~m}$ main mirror, in good agreement with the UVES online documentation. The absolute magnitude is then $M_{5007} \approx 1$ for a distance of $137 \mathrm{kpc}$ (Mackey \& Gilmore 2003). In doing this calculation, it was taken into account that the slit acts as a "shutter" that scans 20 " during each 2400 s exposure, so that the effective integration time for any given point on the sky is $120 \mathrm{~s}$ per scan. The above numbers should be considered orderof-magnitude estimates only, but it does seem clear that this PN is relatively faint, about 6 mag below the tip of the PN luminosity function (Jacoby 1989).

The [O III] line profiles are well-resolved in the UVES spectra. Figure 3 shows the profile of the [O III] $\lambda 5007 \AA$ line, corrected to zero radial velocity using the $\mathrm{H} \beta$ stellar absorption line seen in the GC spectrum. The line profile extends from about $5005.9 \AA$ to $5007.8 \AA$ and is strongly asymmetric, but the midpoint of this interval coincides with the rest wavelength of [O III] (indicated by a vertical dashed line). This further supports the physical association of the source of the [O III] emission with the Fornax dSph and H5. The line half-width corresponds to an expansion velocity of $V_{\mathrm{e}} \approx 55 \mathrm{~km} \mathrm{~s}^{-1}$, which is fairly high for a PN. The median $V_{\mathrm{e}}$ listed by Acker et al. (1992) is $16 \mathrm{~km} \mathrm{~s}^{-1}$, and only 4 of the $284 \mathrm{PNe}$ in their compilation with $V_{\mathrm{e}}$ measurements have $V_{\mathrm{e}}>50 \mathrm{~km} \mathrm{~s}^{-1}$. Data for PNe in other Local Group galaxies confirm that such high expansion velocities are rare and offer some evidence for a correlation of $V_{\mathrm{e}}$ with excitation class, although not with $M_{5007}$ (Richer 2006).

\subsection{Possible identification of the nebula in HST/WFPC2 imaging}

To test whether the source of the [O III] emission seen in the UVES spectra can be identified independently, images from the Wide Field Planetary Camera 2 (WFPC2) on board the Hubble Space Telescope were downloaded from the archive at the Canadian Astronomy Data Centre and inspected. H5 was observed under programme 5917 (P.I. R. Zinn) in the $F 555 W(\approx V)$ and $F 814 W(\approx I)$ filters with exposure times of $5640 \mathrm{~s}$ and $7720 \mathrm{~s}$. The cluster is centred on the PC chip and the co-added images provided by the archive were used directly.

A section of the $F 555 \mathrm{~W}$ image is shown in the left-hand panel of Fig. 4, centred on the location of the emission line source derived from the UVES spectra. The circle has a radius of $1^{\prime \prime}$ and the arrows indicate the orientation ( $\mathrm{N}$ up, $\mathrm{E}$ to the left). The centre of the cluster is near the upper left corner of the panel and identification of the PN counterpart is clearly complicated by severe crowding. Several objects are located near the expected location, but no unique identification of the [O III] source is immediately possible. 


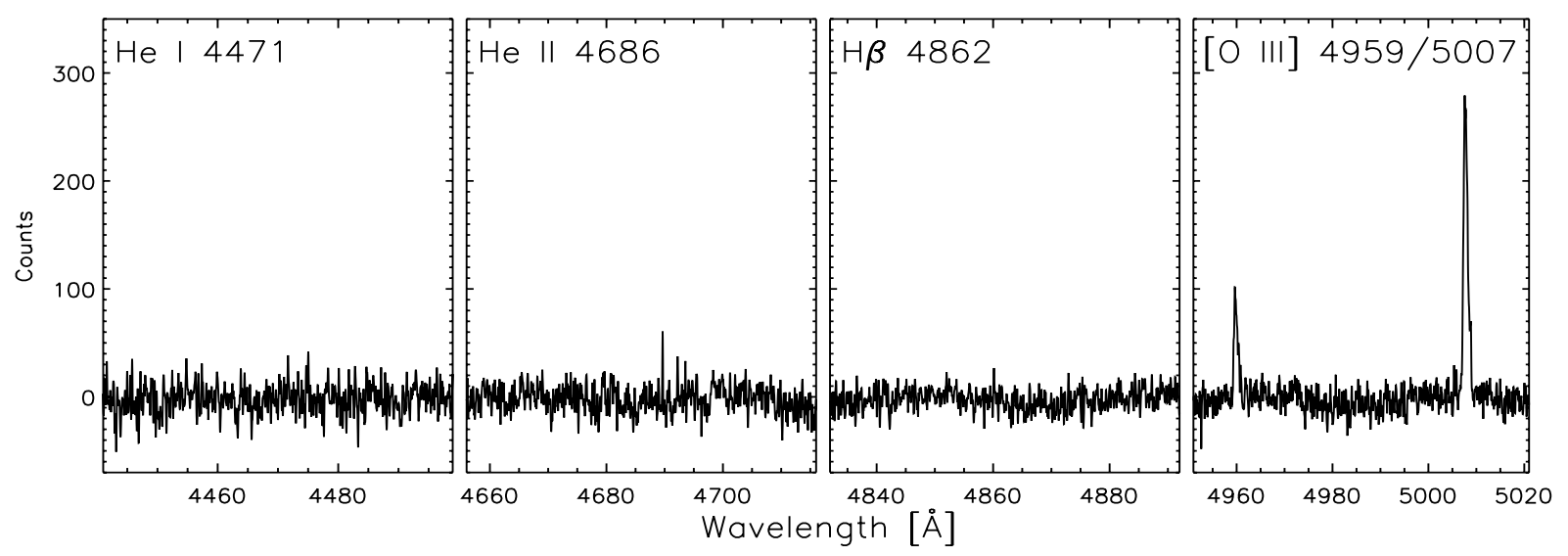

Fig. 2. Spectral regions around He I, He II, H $\beta$, and [O III]. The contribution from GC stars has been subtracted. The spectra have been smoothed with a Gaussian of $F W H M=5$ pixels. Only the [O III] 4959, $5007 \AA$ A lines are detected.

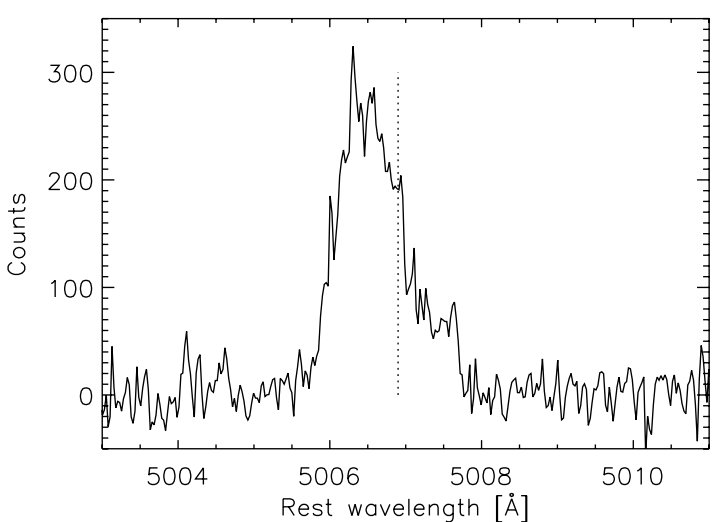

Fig. 3. The [O III] $5007 \AA$ line profile. The contribution from GC stars has been subtracted out and the wavelength scale corrected for the radial velocity of H5. No smoothing has been applied.

The ratio of the $F 555 W$ and $F 814 W$ images is shown in the right-hand panel of Fig. 4. Darker shading indicates a higher ratio of $F 555 W / F 814 W$, i.e. a bluer integrated colour. Due to the more extended point spread function in $F 814 \mathrm{~W}$, stars with red colours tend to appear as white annular structures, while stars with blue colours appear as dark points. Near the centre of the circle (slightly to the southeast) is an extended dark ring, which is identified as the likely counterpart of the [O III] emission. The [O III] lines fall well within the transmission of the $F 555 W$ filter, while no strong emission lines fall within the $F 814 W$ filter, so the nebula is indeed expected to have a blue $F 555 \mathrm{~W} / \mathrm{F} 814 \mathrm{~W}$ colour. Figure 4 hints at a slight asymmetry in the E-W direction, but generally the nebula appears fairly round. The diameter is about 5 WFPC2 pixels, i.e. $0^{\prime} .23$ or $\approx 0.15 \mathrm{pc}$, a fairly typical value for PNe (e.g. Sabbadin et al. 1984). The corresponding expansion age is then about 1300 years.

\section{Discussion and conclusions}

The new PN candidate in $\mathrm{H} 5$ doubles the number of PNe in the Fornax dSph. That it has escaped detection so far, despite many studies of the Fornax GCs, is probably due to its relative faintness and location far enough from the centre $\left(\sim 11^{\prime \prime} 5\right)$ of $\mathrm{H} 5$ to be easily missed in an ordinary long-slit spectrum. As seen in Fig. 4, it is also inconspicuous even in deep (broad-band) HST images, although it should be easily identifiable in narrow-band WFPC2, ACS/HRC, or WFC3 images.
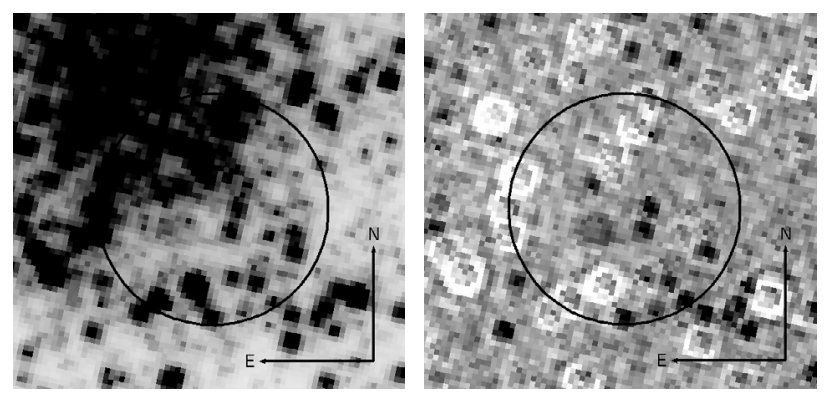

Fig. 4. Left: HST/WFPC2 F555W image of H5. Right: ratio of F555W and $F 814 W$ exposures (black $=$ higher ratio of $F 555 W / F 814 W$ ). The circle $\left(\right.$ diameter $\left.=1^{\prime \prime}\right)$ is centred on the location of the emission line object derived from the spectra (Fig. 1).

As already noted by Danziger et al. (1978), comparison with other Local Group galaxies suggests that about one PN should be expected in the Fornax dSph. More recently, Magrini et al. (2003) concluded from a census of PNe in the Local Group that one PN is expected per $10^{6.92} L_{V} / L_{V, \odot}$. Assuming $M_{V} \approx-13.1$ for Fornax (Demers et al. 1994), 1.7 PNe are then expected. Thus, the discovery of a second candidate does not change the conclusion that the PN population of Fornax is consistent with what is observed in other Local Group galaxies. The Sagittarius $\mathrm{dSph}$, which is somewhat brighter than Fornax, is now known to host four PNe that span a range in metallicity and thus, like those in Fornax, appear to trace different populations (Zijlstra et al. 2006). However, none of these is associated with any of the $\sim 8$ known GCs in the Sagittarius dSph (van den Bergh 2007). What is the likelihood of detecting a PN in one of the Fornax GCs specifically? Clearly, one should be careful about drawing statistical conclusions based on a sample of one object. A naive scaling of the four PNe known in the $\sim 150$ Milky Way GCs (Harris 1996), predicts about 0.13 PNe in the 5 Fornax GCs, but Poisson statistics would still yield $1 \mathrm{PN}$ in a Fornax-like GC system in about $11 \%$ of the cases.

The nebula in H5 is a rather peculiar object. The expansion velocity is high for a PN, although hydrodynamical simulations (Villaver et al. 2002) reproduce the observed combination of radius and $V_{\mathrm{e}}$ at late stages of the PN evolution for a $1 M_{\odot}$ progenitor. There is some other evidence linking high $\mathrm{PN} V_{\mathrm{e}}$ values to old stellar populations (Richer 2006). High $V_{\mathrm{e}}$ values are also observed in bipolar PNe with intermediate-mass progenitors (Corradi \& Schwartz 1995), but the nebula in H5 does not 
appear to belong to this class (Fig. 4). The non-detection of emission lines other than [O III] is even more atypical. In this regard the nebula resembles the peculiar PN IRAS 18333-2357 in the metal-poor Galactic GC M 22, where the absence of emission lines other than [O III] 4959/5007 $\AA$ and [Ne III] 3869/3967 is thought to imply an extreme $\mathrm{H}$ depletion (Cohen \& Gillett 1989). However, IRAS 18333-2357 is exceedingly faint: the [O III] flux is comparable to that of the nebula in H5, although M 22 is a factor of 40 closer! Extreme $\mathrm{H}$ depletion has also been found in knots within some Galactic PNe, e.g. Abell 30 (Wesson et al. 2003). A possible scenario is that this is the result of a late thermal pulse, occurring after most of the H-rich envelope had already been ejected (Iben et al. 1983). Depending on when exactly this happened, an outer low-surface brightness PN might also be present, as in Sakurai's object (Duerbeck \& Benetti 1996).

Further observations of the nebula in H5 might help shed more light on its nature. HST narrow-band imaging might be rewarding, and a comparison of the morphology with that of PNe in Galactic GCs and in the Sagittarius dSph would be interesting, e.g. in relation to differences in the motion with respect to an ambient interstellar/intergalactic medium. The velocity dispersion of the Fornax GC system is only $6 \mathrm{~km} \mathrm{~s}^{-1}$ (Cohen 1983), but Fornax may still be interacting with intergalactic gas in the Local Group (although this point remains uncertain; Piatek et al. 2007). It would also be desirable to search for emission lines other than those from [O III], such as [N II], [Ne III], ionised He, and (of course) the hydrogen Balmer lines.

Acknowledgements. J. Walsh, A. Zijlstra, O. Pols, H. Lamers, and the referee, Dr. L. Magrini, are thanked for several useful suggestions. The abundance study for which the UVES data were obtained is a collaboration with R. Peterson, J. Strader, and J. Brodie of the SAGES group. This research has used the facilities of the Canadian Astronomy Data Centre operated by the National Research Council of Canada with the support of the Canadian Space Agency.

\section{References}

Acker, A., Ochsenbein, F., Stenholm, B., et al. 1992, Strasbourg-ESO Catalogue of Galactic Planetary Nebulae

Battaglia, G., Tolstoy, E., Helmi, A., et al. 2006, A\&A, 459, 423

Buonanno, R., Corsi, C. E., Castellani, M., et al. 1999, AJ, 118, 1671

Cohen, J. 1983, ApJ, 270, L41

Cohen, J. G., \& Gillett, F. C. 1989, ApJ, 346, 803

Corradi, R. L. M., \& Schwartz, H. E. 1995, A\&A, 293, 871

Danziger, I. J., Dopita, M. A., Hawarden, T. G., \& Webster, B. L. 1978, ApJ, 220,458

Demers, S., Irwin, M. J., \& Kunkel, W. E. 1994, AJ, 108, 1648

Dopita, M. A., \& Meatheringham, S. J. 1990, ApJ, 357, 140

Duerbeck, H. W., \& Benetti, S. 1996, ApJ, 468, L111

Grevesse, N., \& Sauval, A. J. 1998, Space Sci. Rev., 85, 161

Harris, W. E. 1996, AJ, 112, 1487

Helmi, A., Irwin, M. J., Tolstoy, E., et al. 2006, ApJ, 651, 121

Hodge, P. W. 1961, AJ, 66, 83

Iben, I., Kaler, J. B., Truran, J. W., \& Renzini, A. 1983, ApJ, 264, 605

Jacoby, G. H. 1989, ApJ, 339, 39

Jacoby, G. H., Morse, J. A., Kellar Fullton, L., Kwitter, K. B., \& Henry, R. B. C. 1997, AJ, 114, 2611

Kniazev, A. Y., Grebel, E. K., Pustilnik, S. A., \& Pramskij, A. G. 2007, A\&A, 468,121

Letarte, B., Hill, V., Jablonka, P., et al. 2006, A\&A, 453, 547

Mackey, A. D., \& Gilmore, G. F. 2003, MNRAS, 340, 175

Magrini, L., Corradi, R. L. M., Greimel, R., et al. 2003, A\&A, 407, 51

Osterbrock, D. E. 1988, Astrophysics of Gaseous Nebulae and Active Galactic Nuclei (Sausalito, CA: University Science Books)

Piatek, S., Pryor, C., Bristow, P., et al. 2007, AJ, 133, 818

Richer, M. G. 2006, in Planetary Nebulae in our Galaxy and Beyond, ed. M. J. Barlow, \& R. H. Méndez, IAU Symp., 234, 317

Sabbadin, F., Bianchini, A., \& Hamzaoglu, E. 1984, A\&A, 136, 200

Schlegel, D. J., Finkbeiner, D. P., \& Davis, M. 1998, ApJ, 500, 525

Stetson, P. B., Hesser, J. E., \& Smecker-Hane, T. A. 1998, PASP, 110, 747

Strader, J., Brodie, J. P., Forbes, D. A., Beasley, M. A., \& Huchra, J. P., 2003, AJ, 125, 1291

van den Bergh, S. 1998, ApJ, 505, L127

van den Bergh, S. 2007, AJ, 134, 344

Villaver, E., Manchado, A., \& García-Segura, G. 2002, ApJ, 581, 1204

Wesson, R., Liu, X.-W., \& Barlow, M. J. 2003, MNRAS, 340, 253

Zijlstra, A. A., Gesicki, K., Walsh, J. R., et al. 2006, MNRAS, 369, 875 